See discussions, stats, and author profiles for this publication at: https://www.researchgate.net/publication/319501777

\title{
An Exact Approach for the Balanced k-Way Partitioning Problem with Weight Constraints and its Application to Sports Team Realignment
}

Article in Journal of Combinatorial Optimization · September 2017

DOI: 10.1007/s10878-018-0254-1

\section{CITATIONS}

4 authors, including:

Diego Recalde

Escuela Politécnica Nacional

7 PUBLICATIONS 36 CITATIONS

SEE PROFILE

Walter Polo Vaca

Escuela Politécnica Nacional

5 PUBLICATIONS 60 CITATIONS

SEE PROFILE
READS

63

Daniel Severin

Rosario National University

22 PUBLICATIONS 52 CITATIONS

SEE PROFILE

Some of the authors of this publication are also working on these related projects:

Project $\quad$ Operations Research in Sports. Methods of Integer Progamming and Combinatorial Optimization View project

Project PROJECT PIJ-15-12 View project 


\title{
An Exact Approach for the Balanced k-Way Partitioning Problem with Weight Constraints and its Application to Sports Team Realignment
}

\author{
Diego Recalde - Daniel Severín . \\ Ramiro Torres • Polo Vaca
}

Received: date / Accepted: date

\begin{abstract}
In this work a balanced $k$-way partitioning problem with weight constraints is defined to model the sports team realignment. Sports teams must be partitioned into a fixed number of groups according to some regulations, where the total distance of the road trips that all teams must travel to play a Double Round Robin Tournament in each group is minimized. Two integer programming formulations for this problem are introduced, and the validity of three families of inequalities associated to the polytope of these formulations is proved. The performance of a tabu search procedure and a Branch \& Cut algorithm, which uses the valid inequalities as cuts, is evaluated over simulated and real-world instances. In particular, an optimal solution for the realignment of the Ecuadorian Football league is reported and the methodology can be suitable adapted for the realignment of other sports leagues.
\end{abstract}

Keywords Integer programming models · Graph partitioning · Tabu search · Sports team realignment.

\footnotetext{
A preliminary version of this paper appeared at ISCO 2016

D. Recalde $\cdot$ R. Torres · P. Vaca

Escuela Politécnica Nacional, Departamento de Matemática, Ladrón de Guevara E11-253, EC170109 Quito, Ecuador.

E-mail: \{diego.recalde, ramiro.torres, polo.vaca\}@epn.edu.ec

D. Recalde

Research Center on Mathematical Modelling (MODEMAT), Escuela Politécnica Nacional, Quito, Ecuador.

D. Severín

FCEIA, Universidad Nacional de Rosario and CONICET, Argentina.

E-mail: daniel@fceia.unr.edu.ar
} 


\section{Introduction}

A fundamental problem in Combinatorial Optimization is to partition a graph in several parts. There are many different versions of graph partitioning problems depending on the number of parts required, the type of weights on the edges or nodes, and the inclusion of several other constraints like restricting the number of nodes in each part. Usually, the objective of these problems is to divide the set of nodes into subsets with a strong internal connectivity and a weak connectivity between them. Most versions of these problems are known to be NP-hard. In this paper, a problem consisting of partitioning a complete or a general graph in a fixed number of subsets of nodes such that their cardinality differ at most in one and the total weight of each subset is bounded, is introduced. The objective aims to minimize the total cost of edges with end-nodes in the same subset. The motivation to state this problem was the realignment of the second category of the Ecuadorian football league.

The sports team realignment deals with partitioning a fixed number of professional sports teams into groups or divisions of similar characteristics, in which a tournament is played. Commonly, a geographical criterion is used to construct the divisions in order to minimize the sum of intra-divisional travel costs. The Ecuadorian football federation (FEF) adapted the last benchmark and imposes that the realignment of the second category of the professional football league must be made by considering provinces instead of teams. Thus, in the realignment, the provinces are divided into four geographical zones. In each zone, two subgroups of teams are randomly constructed by regarding the two best teams of each province, satisfying that two teams of the same province do not belong to the same subgroup, and every subgroup must have the same number of best and second-best teams whenever possible. The eight subgroups play a Double Round Robin Tournament, where the champion of each subgroup and the four second-best teams with the highest scores advance to another stage of the championship. In 2014, FEF managers requested to the authors of this work how to make the realignment of the second category of the Ecuadorian football league in an optimal way, considering 21 provinces and 42 teams to be partitioned in 4 groups. Prior to this requirement, they made a realignment in which the total road travel distance was $39830.4 \mathrm{~km}$. However, the optimal solution reduced this road travel distance in $1532.4 \mathrm{~km}$ with the corresponding highly relevant logistics and economical benefits for the large number of people involved. A graphical representation is depicted in Figure 1. In a preliminary work Recalde et al (2016), the problem was modeled as a $k$-clique partitioning problem with constraints on the sizes and weights of the cliques and formulated as an integer program. Unfortunately, there was an instance related to a proposal to change the design of the realignment that could not be solved to optimality. Consequently, the present paper aims to improve the previous practical results and provide more theoretical insightful about this problem.

The proposal for realignment the second category of the Ecuadorian football league addressed in the previous work, and revisited in this paper, consists 
Empirical solution

Road trip distance: $39830.4 \mathrm{~km}$

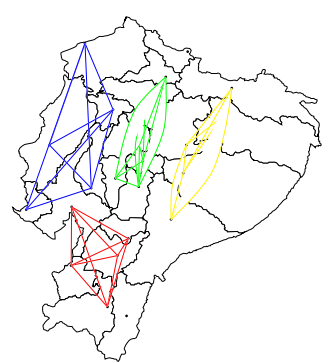

Optimal solution

Road trip distance: $38298 \mathrm{~km}$

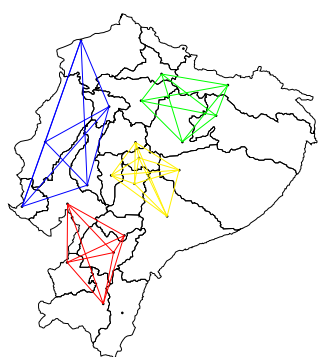

Fig. 1 Empirical vs optimal solution, 2014 edition

of directly making the divisions by using teams instead of provinces, as it is done in other international leagues. During the 2015 season of the second category of the Ecuadorian football league, 44 teams participated (22 provincial associations) and the set of teams was divided into 4 groups with 5 teams and 4 groups with 6 teams. In the context of this problem, the distance between two teams is defined as the road trip distance between the venues of the teams. The strongness or weakness of a team is quantified by means of a parameter that measures its football level considering the historical performance of each team. Thus, the problem studied in this paper consists of partitioning the set of teams into $k$ groups such that the number of teams in each group differs at most by one, there exists a certain homogeneity of football performance among the teams in each class of the partition, and minimizing an objective function corresponding to the total geographical distance intra-group.

The sports team realignment problem has been modeled in different ways and for different leagues in other countries. A quadratic binary programming model is set up to divide 30 teams, of the National Football League (NFL) in the United States, into 6 compact divisions of 5 teams each (Saltzman and Bradford 1996). The results, obtained directly from a nonlinear programming solver, are considerably less expensive for the teams in terms of total intra-divisional travel, in comparison with the realignment of the 1995 edition of this league. On the other hand, McDonald and Pulleyblank (2014) propose a geometric method to construct realignments for several sports leagues in the United States: NHL, MLB, NFL and NBA. The authors claim that with their approach they always find the optimal solution. To prove this, they solve mixed integer programming problems corresponding to practical instances, using CPLEX.

In the case that it is possible to divide the teams into divisions of equal size, this problem can be modeled as a $k$-way equipartition problem: given an undirected graph with $n$ nodes and edge costs, the problem consists of finding a $k$-partition of the set of nodes, each of the same size, such that the total cost of edges which have both endpoints in one of the subsets of the partition is minimized. An example of this case was given by Mitchell (2001), who optimally solved the realignment of the NFL in the United States for 
32 teams and 8 divisions by using a branch-and-cut algorithm. Moreover, the author shows that the 2002 edition of the NFL could have reduced the sum of intra-divisional travel distances by $45 \%$.

When $n \bmod k \neq 0$, the sports team realignment problem can be modeled as a Clique Partitioning Problem (CPP) with constraints in the sizes of the cliques (Ji and Mitchell 2007), as we will see in the next section. The $\mathrm{CPP}$ has been extensively studied in the literature. This graph optimization problem was introduced by Grötschel and Wakabavashi (1989) to formulate a clustering problem. They studied it from a polyhedral point of view and its theoretical results have been used in a cutting plane algorithm that includes heuristic separation routines for some classes of facet-defining inequalities. Ferreira et al (1998) analyzed the problem of partitioning a graph satisfying capacity constraints on the sum of the node weights in each subset of the partition. Jaehn and Pesch (2013) proposed a branch-and-bound algorithm where tighter bounds for each node in the search tree are reported. Additionally, a variant where constraints on the size of cliques are introduced for the CPP is studied by Labbé and Özsov (2010) and a competitive branch-and-cut for a particular problem based on it have been implemented (Catanzaro et al 2011). Regarded to applications of the Graph Partitioning Problem, it is widely known that the canonical application of this problem is the distribution of work to processors of a parallel machine (Hendrickson and Kolda 2000). Other well known applications include VLSI circuit design (Kahng et al 2011), Mobile Wireless Communications (Fairbrother et al 2017) and the already mentioned sports team realignment (Mitchell 2003). For a complete survey of applications and recent advances in graph partitioning, see Buluc et al (2016).

This paper proposes two integer formulations for the $k$-way equipartition problem which are formally defined in Section 2. Moreover, valid inequalities for the polytopes of these formulations are derived in Section 3, which are integrated in a Branch \& Bound \& Cut scheme to solve to optimality instances of 54 teams and, in particular, a hard real-world instance of 44 teams not solved in a previous work. Additionally, a tabu search method for finding feasible high quality solutions is shown in Section 4. In Section 5, the tabu search method and the usage of valid inequalities are integrated in several strategies to solve the real-world as well as the simulated instances. Finally, Section [6] concludes the paper with some remarks.

\section{Problem definition and integer programming formulations}

By associating the venues of teams with the nodes of a graph, the distance between venues with costs on the edges, and football performance of the teams with weights on the nodes, a typical realignment problem can be modeled as a $k$-way partitioning problem with constraints on the size (number of nodes in each subset differs at most in one) and weight of the cliques (total sum of node weights in the clique). From now on, we refer to this problem as a balanced $k$-way partitioning problem with weight constraints $(B k-W W C)$. 
Let $G=(V, E)$ be an undirected complete graph with node set $V=$ $\{1, \ldots, n\}$, edge set $E=\{\{i, j\}: i, j \in V, i \neq j\}$, cost on the edges $d: E \longrightarrow$ $\mathbb{R}^{+}$, weights on nodes $w: V \longrightarrow \mathbb{R}^{+}$and a fixed number $k \geq 2$. A $k$-partition of $G$ is a collection of $k$ subgraphs $\left(V_{1}, E\left(V_{1}\right)\right), \ldots,\left(V_{k}, E\left(V_{k}\right)\right)$ of $G$, where $V_{i} \neq \emptyset$ for all $i=1, \ldots, k, V_{i} \cap V_{j}=\emptyset$ for all $i \neq j, \cup_{i=1}^{k} V_{i}=V$, and $E\left(V_{i}\right)$ is the set of edges with end nodes in $V_{i}$. Note that all subgraphs $\left(V_{i}, E\left(V_{i}\right)\right)$ are cliques since $G$ is a complete graph. Moreover, let $W_{L}, W_{U} \in \mathbb{R}^{+}, W_{L} \leq W_{U}$, be the lower and upper bounds, respectively, for the weight of each clique (which is part of the input of our problem). The weight of a clique is the total sum of the node weights in the clique. Then, $\mathrm{B} k$-WWC consists of finding a $k$-way partition such that

$$
\begin{array}{ll}
W_{L} \leq \sum_{j \in V_{c}} w_{j} \leq W_{U}, & \forall c=1, \ldots, k, \\
|| V_{i}|-| V_{j} \| \leq 1, & \forall i, j=1,2, \ldots, k, \quad i<j,
\end{array}
$$

and the total edge cost over all cliques is minimized. In a previous work (Recalde et al 2016), the NP-hardness of the B $k$-WWC was proved by a polynomial transformation from the 3-Equitable Coloring Problem.

It is known that algorithms based on integer programming techniques are proved to be the best tools for dealing with problems such as $\mathrm{B} k$-WWC. As it was mentioned in the introduction, another CPP was successfully addressed by Catanzaro et al (2011), which used an integer programming formulation for the size-constrained clique partitioning problem given by Labbé and Özsoy (2010). In that formulation, a binary variable is defined for each edge. Let $x_{i j}$ be the variable associated to the edge $\{i, j\}$. Then, $x_{i j}=1$ if nodes $i$ and $j$ belong to the same clique, and $x_{i j}=0$ otherwise. The formulation is stated as follows:

$$
\begin{array}{ll}
\min \sum_{\{i, j\} \in E} d_{i j} x_{i j} & \\
\text { s.t. } & \forall 1 \leq i<j<l \leq n, \\
\quad x_{i j}+x_{j l}-x_{i l} \leq 1, & \forall 1 \leq i<j<l \leq n, \\
x_{i j}-x_{j l}+x_{i l} \leq 1, & \forall 1 \leq i<j<l \leq n, \\
-x_{i j}+x_{j l}+x_{i l} \leq 1, & \forall i \in V, \\
F_{L} \leq 1+\sum_{j:\{i, j\} \in E} x_{i j} \leq F_{U}, & \forall\{i, j\} \in E
\end{array}
$$

The objective function (3) seeks to minimize the total edge cost of the $k$ cliques. Constraints (4), (5), and (6) are the so-called triangle inequalities introduced by Grötschel and Wakabavashi (1989), which guarantee that if three nodes $i, j, l$ of $V_{c}$ are linked by two edges $\{i, j\}$ and $\{j, l\}$, then the third edge $\{i, l\}$ must be also included in the solution, for all $c$. Constraints (7) ensure that the 
cardinality of each clique is between values $F_{L}$ and $F_{U}$, and constraints (8) determine that all variables are binary.

In our case, in order to model the $\mathrm{B} k$-WWC, the cardinality of any two cliques must differ at most in one, i.e., from now on, $F_{L}:=\lfloor n / k\rfloor \geq 2$ and $F_{U}:=\lceil n / k\rceil$. Moreover, additional constraints that impose the weight requirements on each clique are included:

$$
W_{L} \leq w_{i}+\sum_{j:\{i, j\} \in E} w_{j} x_{i j} \leq W_{U}, \quad \forall i \in V,
$$

As the cardinality of each subset in the partition depends on $n$ and $k$, when $k$ divides $n$, the formulation (3)-(9) returns $k$ cliques with exactly $n / k$ nodes and the problem corresponds to the $k$-way equi-partition problem Mitchell (2001). In this case, constraints (7) can be rewritten as:

$$
\sum_{j:\{i, j\} \in E} x_{i j}=\frac{n}{k}-1, \quad \forall i \in V,
$$

When $k$ does not divide $n$, the previous constraints are not enough to guarantee that integer solutions represent partitions of $k$ cliques. In fact, observe that formulation (3)-(9) may admit feasible solutions for different values of $k$; for example, consider as an instance of $\mathrm{B} k$-WWC a complete graph with $n=23$ and $k=7$, which implies that $F_{L}=3$ and $F_{U}=4$. However, a feasible solution for this instance could be a partition consisting of one subset of nodes with cardinality equal to 3 and five subsets with cardinality equal to 4 , which corresponds to a non desired value of $k=6$.

Two alternatives are proposed to overcome this issue. On the one hand, dummy nodes are added to the graph until the condition $n \bmod k=0$ is met, i.e. a set of dummy nodes $\tilde{V}$ of cardinality $k-(n \bmod k)$ is defined. Moreover, for all $i \in \tilde{V}$, costs $d_{i j}=0$ for $j \in V \cup \tilde{V}$, and weights $w_{i}=0$ are fixed. After that, $V$ must be updated with $V \cup \tilde{V}$, and the same for $E$. Finally, observe that two dummy nodes must not be assigned to the same clique in the partition. In order to avoid this impasse, the following constraint is considered:

$$
\sum_{\{i, j\} \subset \tilde{V}} x_{i j}=0
$$

We call $\mathcal{F}_{1}$ to the formulation of $\mathrm{B} k$-WWC composed by the objective function (3) and constraints (44)-(6), (8)-(11).

On the other hand, an alternative to the inclusion of dummy nodes (as formulation $\mathcal{F}_{1}$ states) is to consider a new constraint as follows. Note that in a balanced partition of a graph, there exists $r \in \mathbb{N}$ and $k-r$ disjoint subsets of cardinality $\lceil n / k\rceil$ and $\lfloor n / k\rfloor$, respectively. That is, $n=r F_{U}+(k-r) F_{L}$ where $r=n-k F_{L}=n \bmod k$. Note also that the total number of edges is $r F_{U}\left(F_{U}-1\right) / 2+(k-r) F_{L}\left(F_{L}-1\right) / 2$. Let $\beta_{n, k}$ be the last number. It is easy 
to see that $\beta_{n, k}=\beta_{n, k^{\prime}}$ implies $k=k^{\prime}$, and therefore the following equality forces the partition to have size $k$ :

$$
\sum_{\{i, j\} \in E} x_{i j}=\beta_{n, k}
$$

We call $\mathcal{F}_{2}$ to the formulation composed by the objective function (31) and constraints (4)-(9) and (12).

In some situations, the graph $G$ could be a non-complete one. For example, in real-world instances, there would be an extra requirement where certain pairwise of nodes must not participate in the same clique. This can be modeled by simply deletion of edges $\{u, v\}$ where $u$ and $v$ are those nodes that should be included in different cliques. Such a problem will be known as the generalized balanced $k$-way clique partitioning with weight constraints ( $\mathrm{GB} k$-WWC). The last problem is not harder to solve than $\mathrm{B} k$-WWC. In fact, given an instance of $\mathrm{GB} k$-WWC defined by an arbitrary graph $G=(V, E)$, cost on the edges $d: E \longrightarrow \mathbb{R}^{+}$, weights on nodes $w: V \longrightarrow \mathbb{R}^{+}$, positive numbers $W_{L}$ and $W_{U}$, and a fixed integer number $k \geq 2$, an instance of $\mathrm{B} k$-WWC can be constructed as follows: take a complete graph $G^{\prime}=\left(V^{\prime}, E^{\prime}\right)$ with set of nodes $V^{\prime}=V$, weights $w_{i}^{\prime}=w_{i} \quad \forall i \in V$, numbers $W_{L}^{\prime}=W_{L}, W_{U}^{\prime}=W_{U}, k^{\prime}=k$, and distances

$$
d_{i j}^{\prime}=\left\{\begin{array}{l}
d_{i j}, \text { if }\{i, j\} \in E \\
M, \text { if }\{i, j\} \in E^{\prime} \backslash E
\end{array}\right.
$$

where $M$ is a big number. It is then obvious that $\mathrm{GB} k$-WWC has an optimum solution if and only if the optimum of $\mathrm{B} k$-WWC does not exceed $M$. In practice, one does not have to deal with $M$. As in the case for dummy nodes, considering a constraint $\sum_{\{i, j\} \in E^{\prime} \backslash E} x_{i j}=0$ is enough.

\section{Valid inequalities}

Let $\mathcal{P}$ be the polytope defined by the convex hull of integer solutions of $\mathcal{F}_{1}$ (if $n \bmod k=0$ ) or $\mathcal{F}_{2}$ (otherwise). $\mathcal{P}$ is uniquely determined by the parameters $n, k, W_{L}, W_{U}$ and $w_{i}$ for all $i \in V$. If the weight constraints are redundant, the dimension of this polytope is given by Theorem 3.1 of Labbé and Özsoy (2010) and the equations (10) or (12) are enough to describe the minimal system of $\mathcal{P}$. On the other hand, the weight constraints could make this polytope to be empty. In order to avoid these cases, a necessary condition on weights is established in the following result:

Lemma 1 A necessary condition for the feasibility of $B k-W W C$ is

$$
\max \left\{2,\left\lceil\frac{\sum_{i \in V} w_{i}}{W_{U}}\right\rceil\right\} \leq k \leq \min \left\{\left\lfloor\frac{n}{2}\right\rfloor,\left\lfloor\frac{\sum_{i \in V} w_{i}}{W_{L}}\right\rfloor\right\}
$$


Proof Observe that by assumption $k \geq 2$ and $F_{L} \geq 2$. On the other hand, from constraints (9),

$$
\begin{aligned}
& \sum_{c=1}^{k} W_{L} \leq \sum_{c=1}^{k}\left(w_{i}+\sum_{\{i, j\} \in E} w_{j} x_{i j}\right) \leq \sum_{c=1}^{k} W_{U}, \\
& \sum_{c=1}^{k} W_{L} \leq \sum_{c=1}^{k} \sum_{i \in V_{c}} w_{i} \leq \sum_{c=1}^{k} W_{U}, \\
& k W_{L} \leq \sum_{i \in V} w_{i} \leq k W_{U},
\end{aligned}
$$

from which the result follows.

Observe that $\mathcal{P}$ is contained in the one given by Labbé and Özsoy (2010). Hence, linear relaxations of our formulations can be strengthened by means of known classes of valid and facet-defining inequalities described in previous works. This is the case of the 2-partition inequalities:

$$
\sum_{i \in S} \sum_{j \in T} x_{i j}-\sum_{i_{1}, i_{2} \in S} x_{i_{1} i_{2}}-\sum_{j_{1}, j_{2} \in T} x_{j_{1} j_{2}} \leq|S|,
$$

for every two disjoint nonempty subsets $S, T$ of $V$ such that $|S| \leq|T|$ and $S \cap$ $T=\emptyset$. These inequalities were introduced in the nineties by Grötschel and Wakabavashi (1989) for the Clique Partitioning Polytope and, in recent years, Labbé and Özsoy (2010) explored these inequalities for their polytope. Based on the computational experiments reported in these preceding works, the usage of 2-partition inequalities as cuts evidenced a good behavior and effectiveness in solving the IP model, and they will be considered in the present paper.

In addition, new valid equations and inequalities arise by the introduction of weight constraints (9). Below, three families of valid inequalities for $\mathcal{P}$ are proposed. For any $T \subset V$, define $w(T)=\sum_{i \in T} w_{i}$. Also, for a given $k$-partition $\pi=\left(V_{1}, \ldots, V_{k}\right)$ define $E_{\pi}(T)=\cup_{i=1}^{k} E\left(V_{i} \cap T\right)$. That is, $E_{\pi}(T)$ contains all the edges of $\pi$ with end nodes in $T$. Finally, any integer solution lying in a polyhedron $P$ is called a root of $P$.

Proposition 1 Let $T \subset V$ such that $w(T)>W_{U}$. Then, the following $T$ Weight-Cover inequality is valid for $\mathcal{P}$ :

$$
\sum_{\{i, j\} \in E(T)} x_{i j} \leq \frac{(|T|-1)(|T|-2)}{2} .
$$

Proof Let $x$ be a root of $\mathcal{P}$ representing a $k$-partition $\pi$. The left side of the inequality is $\left|E_{\pi}(T)\right|=\sum_{i=1}^{k}\left|V_{i} \cap T\right|\left(\left|V_{i} \cap T\right|-1\right) / 2$. Since $w(T)>W_{U}$, all nodes in $T$ do not belong to the same clique in $\pi$. That is, $T$ has nodes from two or more cliques in $\pi$ and the largest value of $\left|E_{\pi}(T)\right|$ is reached when $T$ has $|T|-1$ nodes belonging to a clique, say $V_{i_{1}}$, and just one node belonging to another one, say $V_{i_{2}}$. In that case, $\left|E\left(V_{i_{1}}\right)\right|=(|T|-1)(|T|-2) / 2$ and $\left|E\left(V_{i_{2}}\right)\right|=0$. Therefore, $\left|E_{\pi}(T)\right| \leq(|T|-1)(|T|-2) / 2$. 
Corollary 1 Let $\{i, j\} \in E$ such that $w_{i}+w_{j}>W_{U}$. Then, $x_{i j}=0$ is a valid equation of $\mathcal{P}$.

Proposition 2 Let $T \subset V$ such that $w(T)>W_{L},|T| \leq F_{L}$ and $r=w(T)-$ $W_{L}$. Then, for all $i \in T$, the following $(T, i)$-Weight-Lowerbound inequality is valid for $\mathcal{P}$ :

$$
w_{i}+\sum_{j \in T \backslash\{i\}} w_{j} x_{i j}+\sum_{j \in V \backslash T}\left(w_{j}+r\right) x_{i j} \geq w(T) .
$$

Proof Let $x$ be a root of $\mathcal{P}$ representing a $k$-partition $\pi$ and w.l.o.g. suppose that $i \in V_{1}$. The left side of the inequality is $w\left(V_{1}\right)+r\left|V_{1} \backslash T\right|$. If $V_{1} \backslash T=\emptyset$, we have $T=V_{1}$ since $|T| \leq F_{L} \leq\left|V_{1}\right|$, and the inequality is valid. If $V_{1} \backslash T \neq \emptyset$ then $w\left(V_{1}\right)+r\left|V_{1} \backslash T\right| \geq w\left(V_{1}\right)+r \geq W_{L}+r=w(T)$.

Proposition 3 Let $T \subset V$ such that $w(T)<W_{U}, r=W_{U}-w(T)$ and $S=\left\{j \in V \backslash T: w_{j}>r\right\} \neq \emptyset$. Then, for all $i \in T$, the following $(T, i)$-WeightUpperbound inequality is valid for $\mathcal{P}$ :

$$
w_{i}+\sum_{j \in T \backslash\{i\}} w_{j} x_{i j}+\sum_{j \in S}\left(w_{j}-r\right) x_{i j} \leq w(T) .
$$

Proof Let $x$ be a root of $\mathcal{P}$ representing a $k$-partition $\pi$ and w.l.o.g. suppose that $i \in V_{1}$. The left side of the inequality is $w\left(T \cap V_{1}\right)+w\left(S \cap V_{1}\right)-r\left|S \cap V_{1}\right|$. If $S \cap V_{1}=\emptyset$, we have $w\left(T \cap V_{1}\right) \leq w(T)$ and the inequality is valid. If $S \cap V_{1} \neq \emptyset$, then $w\left(T \cap V_{1}\right)+w\left(S \cap V_{1}\right)-r\left|S \cap V_{1}\right| \leq w\left(V_{1}\right)-r\left|S \cap V_{1}\right| \leq W_{U}-r\left|S \cap V_{1}\right| \leq$ $W_{U}-r=w(T)$.

The previous results just give conditions for the inequalities to be valid but we are also interested in finding those inequalities that define faces of high dimension, preferably facets of $\mathcal{P}$, since one can reinforce linear relaxations with them. This fact would require a deeper polyhedral study of $\mathcal{P}$. However, for practical purposes, it is enough to propose necessary conditions that guarantee that faces defined by such inequalities have as many roots as possible (or at least be non-empty). These conditions can be further used for the proper separation of the inequalities involved.

Proposition 4 Let $\mathcal{F}$ be the face defined by a $T$-Weight-Cover inequality. If $\mathcal{F} \neq \emptyset$, then there exists $l \in T$ such that $w(T \backslash\{l\}) \leq W_{U}$.

Proof Let $x$ be a root of $\mathcal{F}$ representing a $k$-partition $\pi$. Since $E_{\pi}(T)=(|T|-$ $1)(|T|-2) / 2$, the partition $\pi$ restricted to $T$ must have two components: a clique of size $|T|-1$ and an isolated node $l$. Denote $T^{\prime}=T \backslash\{l\}$. Clearly, $E_{\pi}(T)=E_{\pi}\left(T^{\prime}\right)$. Now, suppose that $w\left(T^{\prime}\right)>W_{U}$. Hence, the $T^{\prime}$-WeightCover inequality is also valid and we obtain $E_{\pi}(T)=E_{\pi}\left(T^{\prime}\right) \leq(|T|-2)(|T|-$ $3) / 2<(|T|-1)(|T|-2) / 2$ which is absurd.

The previous result suggests that, in order to obtain a good Weight-Cover inequality, we should impose that $w(T \backslash\{l\}) \leq W_{U}$ for all $l \in T$ (i.e. $T$ is "minimal" with respect to the condition $\left.w(T)>W_{U}\right)$. 
Proposition 5 Let $\mathcal{F}$ be the face defined by a $(T, i)$-Weight-Lowerbound inequality. If $\mathcal{F} \neq \emptyset$, then $|T| \geq F_{L}-1$.

Proof Let $x$ be a root of $\mathcal{F}$ representing a $k$-partition $\pi$ and suppose that $i \in V_{1}$. Also, let $s=\left|V_{1} \backslash T\right|$. We have $w\left(V_{1}\right)+r s=w(T)$. Therefore,

$$
s=\frac{w(T)-w\left(V_{1}\right)}{r}=\frac{w(T)-w\left(V_{1}\right)}{w(T)-W_{L}} .
$$

Since $w\left(V_{1}\right) \geq W_{L}$, we have $s \leq 1$. Therefore, $|T| \geq\left|V_{1}\right|-s \geq\left|V_{1}\right|-1 \geq F_{L}-1$.

This result simply suggests to consider only Weight-Lowerbound inequalities such that $|T| \geq F_{L}-1$.

Regarding the Weight-Upperbound inequalities, and for the sake of clarity, the roots of the faces defined by such inequalities are classified in two types. Let $\mathcal{F} \neq \emptyset$ be the face defined by a $(T, i)$-Weight-Upperbound inequality, let $x$ be a root of $\mathcal{F}$ representing a $k$-partition $\pi$ where w.l.o.g. $i \in V_{1}$. If $S \cap V_{1}=\emptyset$, we say that $x$ is of Type 1. Otherwise, $x$ is of Type 2. Now, define $\Pi^{t}$ as the set of roots of $\mathcal{F}$ of Type $t$ where $t \in\{1,2\}$.

Proposition 6 Let $\mathcal{F}$ be the face defined by a $(T, i)$-Weight-Upperbound inequality.

(i) If $\mathcal{F} \cap \Pi^{1} \neq \emptyset$, then $|T| \leq F_{U}$

(ii) If $\mathcal{F} \cap \Pi^{2} \neq \emptyset$, then $|T| \geq F_{L}-1$.

Proof We recall that $w\left((T \cup S) \cap V_{1}\right)-r\left|S \cap V_{1}\right|=w(T)$.

(i) If $S \cap V_{1}=\emptyset$, we obtain $w\left(T \cap V_{1}\right)=w(T)$ implying that $T \subset V_{1}$. Therefore, $|T| \leq\left|V_{1}\right| \leq F_{U}$.

(ii) If $S \cap V_{1} \neq \emptyset$, add $r\left|S \cap V_{1}\right|-W_{U}$ to both sides of the equation. We obtain $w\left((T \cup S) \cap V_{1}\right)-W_{U}=w(T)-W_{U}+r\left|S \cap V_{1}\right|=-r+r\left|S \cap V_{1}\right|=r\left(\left|S \cap V_{1}\right|-1\right)$. Since $\left|S \cap V_{1}\right| \geq 1, w\left((T \cup S) \cap V_{1}\right)-W_{U} \geq 0$ and, therefore, $w\left((T \cup S) \cap V_{1}\right) \geq$ $W_{U}$. On the other hand, $w\left(V_{1}\right) \leq W_{U}$ implying that $w\left((T \cup S) \cap V_{1}\right)=W_{U}$. Hence, $w\left(V_{1}\right)=W_{U}, V_{1} \subset T \cup S$ and $\left|S \cap V_{1}\right|=1$. Let $s$ be the unique element from $S \cap V_{1}$. Then, $V_{1} \backslash\{s\} \subset T$. Therefore, $|T| \geq\left|V_{1}\right|-1 \geq F_{L}-1$.

This result suggests to discard those Weight-Upperbound inequalities such that the condition $F_{L}-1 \leq|T| \leq F_{U}$ does not hold.

\section{Deriving upper bounds: a tabu search}

Consider an optimization problem where, given a graph $G=(V, E)$ and a number $k$, the objective is to obtain a partition $\left(V_{1}, \ldots, V_{k}\right)$ of the set of nodes such that ||$V_{i}|-| V_{j}|| \leq 1$ for all $i \neq j$, and to minimize the number of edges in $\bigcup_{i=1}^{k} E\left(V_{i}\right)$. This problem, called $k$-ECP, is iteratively used by the state-of-the-art tabu search algorithm TABUEQCOL for solving the Equitable Coloring Problem (Méndez Díaz et al 2014; Lai et al 2015).

The $k$-ECP is very related to the problem presented in this paper. In fact, it is a particular case of $\mathrm{B} k$-WWC: simply consider a complete graph 
$G^{\prime}=\left(V, E^{\prime}\right), W_{L}=W_{U}=0, w_{i}=0$ for all $i \in V, d_{i j}=1$ for all $\{i, j\} \in E$ and $d_{i j}=0$ for all $\{i, j\} \in E^{\prime} \backslash E$. In this section, we propose a two-phase algorithm based on TABUEQCOL for solving B $k$-WWC which incorporates an additional mechanism to deal with weights.

Tabu search is a metaheuristic method proposed by Glover et al (1985). Basically, it is a local search algorithm which is equipped with additional mechanisms that prevent from visiting a solution twice and getting stuck in a local optimum. The design of a tabu search algorithm involves to define the search space of feasible solutions, an objective function, the neighborhood of each solution, the stopping criterion, the aspiration criterion, the features and how to choose one of them to be stored in the tabu list and how to compute the tabu tenure. The reader is referred to the work by Méndez Díaz et al (2014) for the definitions of these concepts and how to denote them.

Below, the details of our algorithm are presented:

- Search space of solutions. A solution $s$ is a partition $\left(V_{1}, \ldots, V_{k}\right)$ of the set of nodes such that ||$V_{i}|-| V_{j}|| \leq 1$ for all $i \neq j$. For the sake of efficiency, solutions are stored in memory as tuples $\left(V_{1}, \ldots, V_{k}, W_{1}, \ldots, W_{k}, R^{+}, R^{-}\right)$ where $W_{i}=\sum_{v \in V_{i}} w_{v}, R^{+}=\left\{i:\left|V_{i}\right|=\lfloor n / k\rfloor+1\right\}$ and $R^{-}=\left\{i:\left|V_{i}\right|=\right.$ $\lfloor n / k\rfloor\}$.

- Objective function. For a given solution $s$, let $d(s)$ be the sum of the distances of every edge in $E\left(V_{i}\right)$ for all $i \in\{1, \ldots, k\}$ and $I(s)=\left\{i: W_{i}<\right.$ $\left.W_{L} \vee W_{i}>W_{U}\right\}$. The objective function is defined as $f(s)=d(s)+M|I(s)|$ where $M$ is a big value. Note that solutions satisfying $I(s) \neq \varnothing$ are feasible but penalized in $f(s)$.

- Stopping criterion. The algorithm stops when a maximum number of iterations is reached.

- Aspiration criterion. Let $s$ be the current solution and $s^{*}$ be the best known solution so far. When $f(s)<f\left(s^{*}\right), s$ replaces $s^{*}$.

- Set of features. A solution $s$ presents a feature $(v, i)$ if and only if $v \in V_{i}$.

- Initial solution. For all $i \in\{1, \ldots, k\}$, do $V_{i}=\left\{v_{j}:(j-1) \bmod k=i-1\right\}$.

- Neighborhood of a solution. For a given solution $s=\left(V_{1}, \ldots, V_{k}, W_{1}, \ldots, W_{k}\right.$, $\left.R^{+}, R^{-}\right)$, a neighbor $s^{\prime}=\left(V_{1}^{\prime}, \ldots, V_{k}^{\prime}, W_{1}^{\prime}, \ldots, W_{k}^{\prime}, R^{\prime+}, R^{\prime-}\right)$ of $s$ is generated with two schemes:

- 1-move (only applicable when $n$ does not divide $k$ ). For a given $v \in V_{i}$ such that $i \in R^{+}$and a given $j \in R^{-}$, consider $s^{\prime}$ such that node $v$ is moved from $V_{i}$ to $V_{j}$. Formally, $V_{j}^{\prime}=V_{j} \cup\{v\}, W_{j}^{\prime}=W_{j}+w_{v}$, $V_{i}^{\prime}=V_{i} \backslash\{v\}, W_{i}^{\prime}=W_{i}-w_{v}, V_{l}^{\prime}=V_{l}$ and $W_{l}^{\prime}=W_{l}$ for all $l \in$ $\{1, \ldots, k\} \backslash\{i, j\}, R^{\prime+}=R^{+} \cup\{j\} \backslash\{i\}$ and $R^{\prime-}=R^{-} \cup\{i\} \backslash\{j\}$.

- 2-exchange. For a given $v \in V_{i}$ and $u \in V_{j}$ such that $i<j$, consider $s^{\prime}$ such that $v$ is moved to $V_{j}$ and $u$ is moved to $V_{i}$. Formally, $V_{j}^{\prime}=$ $\left(V_{j} \backslash\{u\}\right) \cup\{v\}, W_{j}^{\prime}=W_{j}-w_{u}+w_{v}, V_{i}^{\prime}=\left(V_{i} \backslash\{v\}\right) \cup\{u\}, W_{i}^{\prime}=$ $W_{i}-w_{v}+w_{u}, V_{l}^{\prime}=V_{l}$ and $W_{l}^{\prime}=W_{l}$ for all $l \in\{1, \ldots, k\} \backslash\{i, j\}$, $R^{\prime+}=R^{+}$and $R^{\prime-}=R^{-}$.

- Selection of a feature to add in the tabu list. Once a movement from $s$ to $s^{\prime}$ is performed, $(v, i)$ is stored on the tabu list. 
- Tabu tenure. Once an element is added to the tabu list, it remains there for the next $t$ iterations, where $t$ is an integer randomly generated with a uniform distribution (one of the criteria used by Lai et al (2015)).

Since one pretends the algorithm to be as fast as possible, the value of $f\left(s^{\prime}\right)$ should be computed by adding or subtracting the corresponding difference to $f(s)$. Also, $M$ should not be too high in order to avoid round-off errors. In our case, $M$ was set to 10000 .

A difference between TABUEQCOL and our algorithm is that we are interested in feasible solutions for the $\mathrm{B} k$-WCC but TABUEQCOL does not contemplate weight constraints. For that reason, the entire process is divided in two stages. The first one consists of searching a solution $s$ with $I(s)=\varnothing$ while the second one is focused on minimizing $d(s)$. We observed that, during the first stage, the search needs to be more diversified. Therefore, different range of values for the tabu tenure in each stage are used. If $f(s) \geq M$ (1st. stage) then $t \in[5,40]$ and if $f(s)<M$ (2nd. stage) then $t \in[5,20]$.

This method can also be used for obtaining feasible solutions of GB $k$ WWC: simply consider $d_{i j}=M$ for those edges $\{i, j\} \notin E$; if $f(s)<M$ then $s$ is a feasible solution of GBk-WWC. However, if the density of edges in the graph $G$ is not high, it would be convenient to exploit the structure of $G$ in the computation of the neighborhood of a solution and tabu tenure, as in the case of TABUEQCOL.

\section{Computational experiments}

In this section, some computational experiments are presented. They consist of comparing different ways of solving the B $k$-WWC, called Strategies and denoted by $\mathcal{S}$. Comparisons are carried out over random instances and, at the end, the resolution of a real-world instance is addressed. The improvement in the results are shown incrementally. That is, each strategy outperforms the previous one. All the experiments were carried out on a machine equipped with an Intel i5 $2.67 \mathrm{GHz}, 4 \mathrm{~Gb}$ of memory, the operating system Ubuntu 16.4 and the solver GuRoBi 6.5. All instances as well as the source code of the implementation can be downloaded from:

http://www.fceia.unr.edu.ar/ daniel/stuff/football.zip

Random instances were generated by computing the coordinates $(x, y)$ of $n$ points with an uniform distribution in the domain $[-100,100] \times[-100,100]$. Then, for every pair of points $i, j, d_{i j}$ is assigned the euclidean distance between both points. Weights $w_{i}$ are random values generated with a uniform distribution in the range $[0.1,0.9]$, and $W_{L}=\mu(n / k)-\sigma, W_{U}=\mu(n / k)+\sigma$ where $\mu$ and $\sigma$ are the average and standard deviation of the weights of all points. Combinations of $n$ are $k$ were chosen so that $k$ does not divide $n$, similar to those values of real instances.

Results of the experiments are summarized in Tables 2 to 6. whose format are as follows: first and second columns display the number of the instance 
and its optimal value, and remaining columns show the number of $B \& B$ nodes evaluated and the time elapsed in seconds of the optimization. A time limit of one hour is imposed. For those instances that are not solved within this limit, the gap percentage between the best lower and upper bound found is reported. Last row displays the average over all instances, except for Tables 4 and 6 (marked with a dagger) where it shows the average over those instances solved by all strategies being compared (i.e. 21, 23, 24, 26, 28, 29 and 30 for Table 4. 42, 43, 45, 47, 49 and 50 for Table 6). Boldface indicates the best results.

$\mathcal{F}_{1}$ (with dummy nodes) vs. $\mathcal{F}_{2}$. Strategy $1\left(\mathcal{S}_{1}\right)$ consists of the resolution of $\mathcal{F}_{1}$, after addition of $k-(n \bmod k)$ dummy nodes, while $\mathcal{S}_{2}$ is the direct resolution of $\mathcal{F}_{2}$. Both use GuRoBi at its default settings. Instead of using a single constraint, such as (11), variables $x_{i j}$ are directly fixed to zero in a straightforward manner. In fact, there are three cases where $x_{i j}$ are set to zero:

$-i, j \in \tilde{V}$ (only when dummy nodes are present, see constraint (11)).

- $\{i, j\} \in E^{\prime} \backslash E$ (only when $G$ is not complete).

- $w_{i}+w_{j}>W_{U}$ (see Corollary 1).

Note that, according to the results reported in the tables, $\mathcal{S}_{2}$ performs better than $\mathcal{S}_{1}$ for larger instances. In particular, $\mathcal{S}_{2}$ solves instance 27 to optimality and reports better gaps than $\mathcal{S}_{1}$ for instances 22 and 25.

Tabu search vs. GuRoBi built-in heuristics. The next step is to use the tabu search method proposed in the previous section. This metaheuristic generates good initial solutions. In particular, it gives the optimal one for almost all instances given in the tables in a reasonable amount of iterations (the unique exception was instance 19 where it could not reach the optimal solution after 1000000 iterations, for two different seeds). Based on experimentation with several random instances and initial seeds, we obtained a formula by linear regression for the limit in the number of iterations needed:

$$
i t_{\text {limit }}=\left\lfloor e^{0.26571 n-0.052978}\right\rfloor
$$

Now, in $\mathcal{S}_{3}, i t_{\text {limit }}$ iterations of the tabu search are executed and the best solution found is provided as an initial integer solution to GuRoBi. Then, $\mathcal{F}_{2}$ is solved with GuRoBi primal heuristics turned off (Heuristics, PumpPasses and RINS parameters are set to zero). In order to make a fair comparison, time reported in tables includes time spent by tabu search. Clearly, $\mathcal{S}_{3}$ outperforms $\mathcal{S}_{2}$. In particular, $\mathcal{S}_{3}$ was able to solve instance 25 by optimality and presents a lower gap than $\mathcal{S}_{2}$ for instance 22 .

Addition of triangular inequalities on demand. Formulation $\mathcal{F}_{2}$ (and also $\mathcal{F}_{1}$ ) has a $O\left(n^{3}\right)$ number of constraints due to triangular inequalities (precisely, $n(n-1)(n-2) / 2)$. Although in presence of variables set to zero some of them become redundant and one can omit them when performing the initial relaxation (e.g. for a given $i<j<l$, if $x_{i j}=0$ then inequalities (4) and (5) are redundant but (6) is not), its number is still high. Since the number of variables 
is $O\left(n^{2}\right)$ it is expected that several triangular inequalities are not active in the fractional solution of the initial relaxation. We noticed that there exists a relationship between being active and the distances of positive variables in its support: the lower the value is $d_{i j}+d_{j l}$, the higher the probability of the inequality $x_{i j}+x_{j l}-x_{i l} \leq 1$ is active. The following experiment reveals this relationship.

Let $\mathcal{T}$ be the set of triangular inequalities and let $\tilde{d}(t)$ be a value assigned to each $t \in \mathcal{T}$ as follows:

$$
\tilde{d}(t)= \begin{cases}d_{i j}+d_{j l}, & \text { when } t \text { is a constr. (4), } \\ d_{i j}+d_{i l}, & \text { when } t \text { is a constr. (5) }, \\ d_{j l}+d_{i l}, & \text { when } t \text { is a constr. (6). }\end{cases}
$$

We first order all triangular inequalities according to the value $\tilde{d}(t)$ from lowest to highest and we make an equitable partition $I_{1}, I_{2}, \ldots, I_{10}$ of the set $\mathcal{T}$ in 10 deciles. That is, $\cup_{r=1}^{10} I_{r}=\mathcal{T}$ and $\left|I_{r}\right|=\left\lceil\frac{n+r-10}{10}\right\rceil$ for all $r=1, \ldots, 10$ where $d(t) \leq d\left(t^{\prime}\right)$ for $t \in I_{r}$ and $t^{\prime} \in I_{r+1}$. Then, we solve $\mathcal{F}_{2}$ without these inequalities in its initial relaxation and, whenever GuRobi obtains a solution (fractional or integer) violating some of them, they are added to the current relaxation: if the solution is fractional, they are added as cuts and if the solution is integer, they are added as lazy constraints. Histograms with the averages (over 10 instances of 44 nodes, each instance having $|\mathcal{T}|=39732$ ) of percentages of triangular inequalities generated per decile $I_{r}$ are shown in Figures 2 and 3 . In the former, only those inequalities added at root node of $B \& B$ tree are considered. In Figure 3, all inequalities are counted. In particular, if the same inequality is generated in two different nodes, it is counted twice.

Note that, at root node, most of the inequalities from $I_{1}$ and $I_{2}$ are generated. In addition, during the $\mathrm{B} \& \mathrm{~B}$ process, inequalities from $I_{8}, I_{9}$ and $I_{10}$ are rarely violated. Based on these observations and additional experimentation, we define the strategy $\mathcal{S}_{4}$ as $\mathcal{S}_{3}$ with the following differences:

- Only inequalities from $I_{1}$ and $I_{2}$ are considered in the initial relaxation.

- Each time an integer solution is found, inequalities from $I_{r}$ with $r \in$ $\{3, \ldots, 10\}$ are checked and violated ones are added as lazy constraints.

- Each time a fractional solution is found, inequalities from $I_{r}$ with $r \in$ $\{3, \ldots, m\}$ are checked and those that are violated by at least 0.1 units, are added as cuts. If the current node is root, $m=10$, otherwise $m=7$.

- Some GuRoBi cuts are disabled (Clique, FlowCover, FlowPath, Implied, MIPSep, Network and ModK).

Observe that $\mathcal{S}_{4}$ behaves much better than $\mathcal{S}_{3}$. This strategy needs less than the half of time used by the preceding one. In addition, it can solve instance 22 to optimality.

Separation of valid inequalities. Here, we experiment with additional custom separation routines embedded in our code, where 2-partition inequalities and the new families of valid inequalities presented in Section 3 are considered. Two experiments are carried out, detailed below. 
Experiment 1: In this experiment, we analyze the effectiveness in terms of reduction in the number of B\&B nodes when Weight-Cover, Weight-Lowerbound and Weight-Upperbound inequalities are used. We also gather helpful information that is further used for the design of the separation routines. For each family, random instances of 44 nodes are solved and, during the optimization, the inequalities satisfying the conditions given in Section 3 are enumerated exhaustively and added when they are violated by an amount of at least $1 \%$ of the r.h.s.

Regarding Weight-Cover inequalities, we restrict the enumeration to $|T| \in$ $\{4,5\}$ since for $|T|=3$ or $|T| \geq 6$ they are seldom violated. Regarding WeightUpperbound inequalities, we impose an additional limit of 1500 nodes since its enumeration in each node consumes a fairly long time. This limit is reached on instances 22,25 and 27 .

Table 1 reports the total number of cuts generated and the number of B\&B nodes evaluated for each family of valid inequalities, and the relative gap when 1500 nodes are reached (only for Weight-Upperbound). The three columns entitled "only GuRoBi" display the same parameters (number of cuts, B\&B nodes and relative gap at 1500 nodes) generated by strategy $\mathcal{S}_{4}$. The last three rows show the averages over all instances, the averages over instances 21, 23, $24,26,28,29$ and 30 (marked with a dagger), and the average of gap over instances 22, 25 and 27 (marked with a double dagger).

We conclude that the addition of Weight-Cover and Weight-Upperbound cuts make a substantial reduction in the number of nodes, whereas Weight-Lowerbound cuts occur less frequently and the reduction in the number of nodes is marginal. We also noticed that violated Weight-Cover inequalities are usually composed of nodes $i$ with high values of the expression $w_{i}+\sum_{j \in V \backslash\{i\}} w_{j} x_{i j}^{*}$, where $x^{*}$ is the current fractional solution. However, violated Weight-Upperbound inequalities do not seem to have an obvious structure that can be exploited. We only consider Weight-Cover inequalities in the next experiment.

Experiment 2: The goal is to compare the performance of $\mathcal{S}_{4}$ when different combinations of separation routines are used. For each combination, random instances of 48 and 54 nodes are solved (see Tables 5 and 6). The execution of these routines is performed only when no triangular inequalities were generated for the current fractional solution, denoted by $x^{*}$. In particular, the separation of 2-partition inequalities is based on the procedure given by Grötschel and Wakabavashi (1989) and Catanzaro et al (2011).

- 2-partition. The following procedure is repeated for each $v \in V$. First, compute $W:=\left\{u \in V \backslash\{v\}: x_{u v}^{*} \notin \mathbb{Z}\right\}$. If $|W| \leq 4$, then stop. Otherwise, set $F:=\emptyset$ and repeat the following 5 times, whenever possible. Pick two random nodes $i, j$ from $W \backslash F$ and set $T:=\{i, j\}$. Keep picking nodes $r$ from $W \backslash F$ such that $x_{r v}^{*}-\sum_{t \in T} x_{r t}^{*}>0$ and add $r$ to $T$ until no more nodes are found. Then, check if the 2-partition inequality (13) with $S=\{v\}$ and $T$ is violated and, in that case, add it as a cut. Finally, make $F:=F \cup T$ (even when the inequality is not violated). The set $F$ (of "forbidden nodes") prevents from generating cuts with similar support. 
- Weight-Cover. First, order nodes $i \in V$ according to the value $q^{*}(i):=$ $w_{i}+\sum_{j \in V \backslash\{i\}} w_{j} x_{i j}^{*}$ from highest to lowest, i.e. $V=\left\{v_{1}, v_{2}, \ldots, v_{n}\right\}$ such that $q^{*}\left(v_{j}\right) \geq q^{*}\left(v_{j+1}\right)$ for all $j$. For each $t \in\{4,5\}$ do the following. Consider every $T$ composed of $t-2$ nodes from $\left\{v_{1}, v_{2}, \ldots, v_{t+2}\right\}$ and 2 more nodes from $V$ (note that $|T|=t$ and the procedure explores $O\left(n^{2}\right)$ combinations). If $w(T)>W_{U}$ and $w(T \backslash\{l\}) \leq W_{U}$ for all $l \in T$, check the $T$-Weight-Cover inequality. If it is violated, add it as a cut.

In the given procedures, an inequality is considered violated if the amount of violation is at least 0.1 . As one can see from the tables, 2-partition together with Weight-Cover cuts is the best choice. We define the strategy $\mathcal{S}_{5}$ as $\mathcal{S}_{4}$ with both separation routines enabled.

Resolution of a real-world instance. As mentioned in the introduction, in the zonal stage of the second category of the Ecuadorian football league, a championship including the two best teams of each provincial associations is played. The set of teams must be partitioned in 8 groups to play a Round Robin Tournament in each one of them. A regulation imposes that two teams of the same provincial association must not belong to the same group. During the 2015 season, 44 teams (22 provincial associations) participated in the tournament, and they were divided in 4 groups of 5 teams and 4 groups of 6 teams.

The nodes of a graph are associated with the venues of the teams. We denote the nodes by $2 i$ and $2 i+1$, corresponding to the venues of the best two teams of each provincial association $i$, for all $i=0,1, \ldots, 21$, and edge $\{i, j\}$ is included if and only if the teams associated to nodes $i, j$ could potencially belong to the same group. In order to satisfy the regulation mentioned before, edges of the form $\{2 i, 2 i+1\}$ do not appear in the graph. Thus, our realignment instance consists of 44 teams which must be partitioned in 8 groups, and the graph $G=(V, E)$ is a particular complement of a matching, i.e. $V=$ $\{0, \ldots, 43\}$ and $E=\{\{i, j\}: 0 \leq i<j \leq 43\} \backslash\{\{2 i, 2 i+1\}: 0 \leq i \leq 21\}$.

For solving our instance, we made a preliminary test of our two best strategies (i.e. $\mathcal{S}_{4}$ vs. $\mathcal{S}_{5}$ ). A time limit of one hour was imposed to them. None of them was able to solve the instance within this limit, but the relative gap reported was $3.22 \%$ for $\mathcal{S}_{4}$ against $2.36 \%$ for $\mathcal{S}_{5}$. Hence, $\mathcal{S}_{5}$ was chosen for solving the instance without time limit. Below, we resume some highlights about the optimization:

- Instance: $|V|=44, k=8, W_{L}=2.08412$ and $W_{U}=4.14835$.

- Iterations performed/time spent by tabu search: 113352 iterations (6.8 sec.).

- Variables and constraints of the initial relaxation: 913 vars., 7444 constr.

- Cuts generated: Gomory (18), Cover (65), MIR (1620), GUB cover (98), Zero-half (1278), triangular (1935), 2-partition (3965), Weight-Cover (14).

- Nodes explored and total time elapsed: 34573 (68374 sec.).

- Optimal value: $21523 \mathrm{~km}$, found by tabu search at iter. 3549 (0.21 sec.).

- Gap evolution: $2.36 \%$ after 1 hour, $1.08 \%$ after 4 hours. 
Since a Double Round Robin Tournament is considered, the total distance is $86092 \mathrm{~km}$. In contrast, the best solution found in our previous work (Recalde et al 2016) had $86192 \mathrm{~km}$ and the gap reported was $12.6 \%$ after 4 hours of execution.

\section{Conclusion and future work}

In this paper, a balanced $k$-way partitioning problem with weight constraints is defined. The problem consists in partitioning a complete or a general graph in a fixed number of subsets of nodes such that their cardinality differs at most in one and the total weight of each subset is bounded. The objective aims to minimize the total cost of edges with end-nodes in the same subset. The problem was formulated as an integer program and several strategies based on exact and methaheuristic methods are evaluated. The motivation to state, formulate and solve this problem was the realignment of the second category of the Ecuadorian football league. The solution of this case of study is based on real world data and the methodology may be suitable applied to the realignment of other sports leagues with similar characteristics.

In order to solve the problem, one of the key decisions was to use a modification of a state-of-the-art tabu search for obtaining good feasible solutions. In particular, our algorithm found the optimal solution of the real-world instance in less than a second, whereas the previous approach given by Recalde et al (2016) was unable to obtain it within 4 hours of CPU time. Another key decision was to include a portion of triangular inequalities $(20 \%$ of them under a specific ordering) in the initial relaxation and manage the remaining ones as cuts or lazy constraints. These facts, in conjunction with other minor decisions, allowed to solve comfortably random instances of 48 nodes in less than half an hour and the real-world one in 19 hours (here, almost all the time was spent on certifying the optimality).

In addition, two formulations are presented and one of them $\left(\mathcal{F}_{2}\right)$ is chosen based on experimentation. A possible cause of the poor performance of $\mathcal{F}_{1}$ could be the existence of symmetrical solutions due to the indistinguishability of dummy nodes. For example, an addition of 4 dummy nodes implies that, for each integer solution of $\mathcal{F}_{2}$, there are 24 symmetrical integer solutions in $\mathcal{F}_{1}$.

We also proposed three families of valid inequalities and two of them have proven to be very effective for reducing the number of B\&B nodes, when they are used as cuts. One of them (Weight-Cover) in conjunction with the well-known 2-partition inequalities, allows to shorten the CPU time in 51\% for $n=48$ (see Table 5) and $56 \%$ for $n=54$ (see Table 6). Moreover, it is able to solve one more instance (48) and the gap reported for those instances not solved in one hour $(41,44,46)$ is smaller. As other state-of-the-art exact algorithms for the $k$-way graph partitioning problem Fairbrother et al (2017); Anjos et al (2013), the best strategies provided here attain optimal solutions for graphs that have around 50 nodes. 
A future work could be to include a separation routine of Weight-Upperbound inequalities and to explore other valid inequalities (for example, by adapting those ones presented by Catanzaro et al (2011)). Finally, at the theoretical level, it could be useful to make a polyhedral study of $\mathcal{P}$, the convex hull of integer solutions of $\mathcal{F}_{2}$, and to propose facet-defining inequalities that can be used as cuts.

Acknowledgements This research was partially supported by the 15-MathAmSud-06 "PACK-COVER: Packing and covering, structural aspects" trilateral cooperation project.

\section{References}

Anjos M, Ghaddar B, Hupp L, Liers F, Wiegele A (2013) Solving k-way graph partitioning problems to optimality: The impact of semidefinite relaxations and the bundle method". In: Jünger M, Reinelt G (eds) Facets of Combinatorial Optimization: Festschrift for Martin Grötschel, Springer Berlin Heidelberg, Berlin, Heidelberg, pp 355-386

Buluç A, Meyerhenke H, Safro I, Sanders P, Schulz C (2016) Recent advances in graph partitioning. In: Kliemann L, Sanders P (eds) Algorithm Engineering: Selected Results and Surveys, Springer International Publishing, Cham, pp 117-158

Catanzaro D, Gourdinb E, Labbé M, Özsoy FA (2011) A branch-and-cut algorithm for the partitioning-hub location-routing problem. Comput Oper Res 38(2):539-549

Fairbrother J, Letchford A, Briggs K (2017) A two-level graph partitioning problem arising in mobile wireless communications. Discr Optim (accepted paper)

Ferreira C, Martin A, de Souza C, Weismantel R, Wolsey L (1998) The node capacitated graph partitioning problem: A computational study. Math Program 81:229-256

Glover F, McMillan C, Novick B (1985) Interactive decision software and computer graphics for architectural and space planning. Ann Oper Res 5(3):557-573

Grötschel M, Wakabayashi Y (1989) A cutting plane algorithm for a clustering problem. Math Program 45:59-96

Hendrickson B, Kolda TG (2000) Graph partitioning models for parallel computing. Parallel Computing 26(12):1519-1534

Jaehn F, Pesch E (2013) New bounds and constraint propagation techniques for the clique partitioning problem. Discr Appl Math 161:2025-2037

Ji X, Mitchell JE (2007) Branch-and-price-and-cut on the clique partitioning problem with minimum clique size requirement. Discr Optim 4:87-102

Kahng A, Lienig J, Markov I, Hu J (2011) VLSI Physical Design: From Graph Partitioning to Timing Closure, 1st edn. Springer Publishing Company, Incorporated

Labbé M, Özsoy FA (2010) Size-constrained graph partitioning polytopes. Discr Math 310:3473-3493

Lai X, Hao JK, Glover F (2015) Backtracking based iterated tabu search for equitable coloring. Eng Appl Artif Intell 46:269-278

McDonald B, Pulleyblank W (2014) Realignment in the NHL, MLB, NFL, and NBA. Journal of Quantitative Analysis in Sports 10(2):225-240

Méndez Díaz I, Nasini G, Severin D (2014) A tabu search heuristic for the equitable coloring problem. Lect Notes Comput Sc 8596:347-358

Mitchell JE (2001) Branch-and-cut for the k-way equipartition problem. Technical report, Department of Mathematical Sciences, Rensselaer Polytechnic Institute

Mitchell JE (2003) Realignment in the national football league: Did they do it right? Naval Research Logistics (NRL) 50(7):683-701

Recalde D, Severin D, Torres R, Vaca P (2016) Balanced partition of a graph for football team realignment in ecuador. Lect Notes Comput Sc 9849:357-368

Saltzman R, Bradford RM (1996) Optimal realignments of the teams in the national football league. Eur J Oper Res 93(3):469-475 


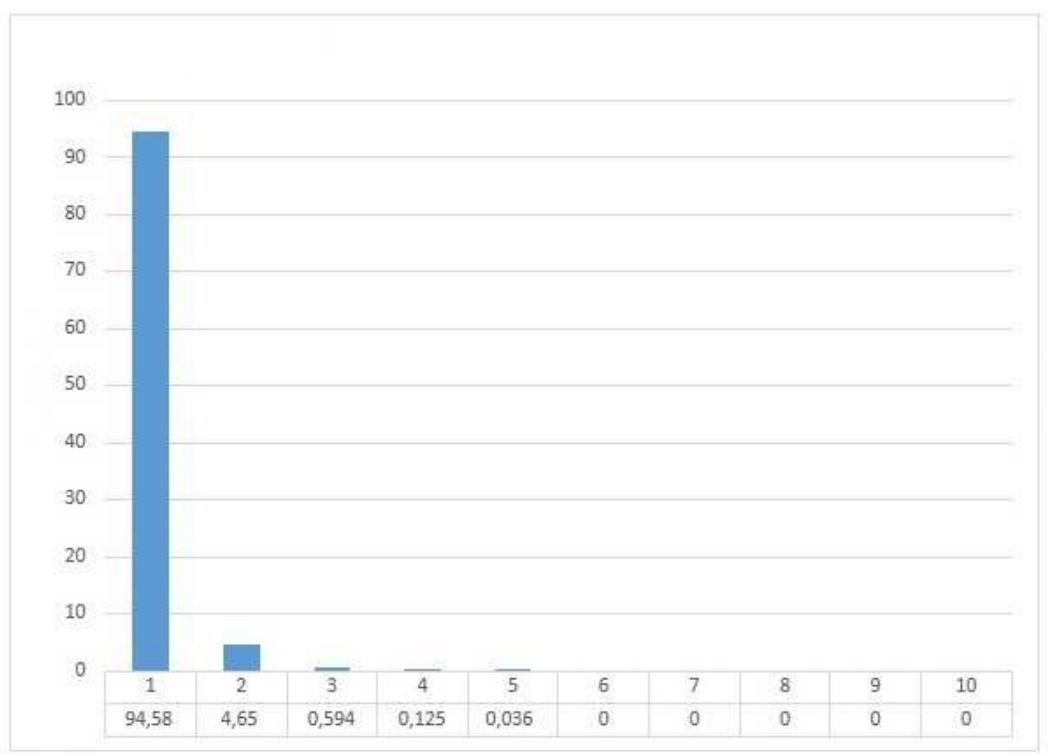

Fig. 2 Average of $\%$ of triangular inequalities added at root node

Table 1 Exhaustive generation of new cuts $(n=44$ and $k=8)$

\begin{tabular}{|c|ccc||cc|cc|ccc|}
\hline & \multicolumn{3}{|c||}{ only GuRoBi } & \multicolumn{2}{c|}{ Weight-Cover } & \multicolumn{2}{c|}{ W.-Lowerb. } & \multicolumn{2}{c|}{ Weight-Upperbound } \\
N. & \multicolumn{2}{|c||}{ Nodes } & Cuts Gap1500 & Nodes & Cuts & \multicolumn{2}{c|}{ Nodes } & Cuts & \multicolumn{2}{c|}{ Nodes Cuts Gap1500 } \\
\hline 21 & 322 & 280 & & 255 & 34 & 281 & 17 & 415 & 58 & \\
22 & 11037 & 782 & $4.71 \%$ & 10290 & 146 & 12595 & 46 & & & $3.58 \%$ \\
23 & 545 & 365 & & 519 & 69 & 629 & 16 & 592 & 208 & \\
24 & 1492 & 576 & & 1023 & 82 & 1465 & 27 & 1427 & 123 & \\
25 & 5413 & 691 & $3.65 \%$ & 6882 & 182 & 6126 & 120 & & & $4.10 \%$ \\
26 & 574 & 379 & & 317 & 36 & 584 & 5 & 665 & 149 & \\
27 & 5360 & 555 & $5.52 \%$ & 3120 & 148 & 5219 & 98 & & & $4.34 \%$ \\
28 & 50 & 84 & & 24 & 25 & 50 & 0 & 29 & 20 & \\
29 & 1518 & 407 & & 659 & 37 & 1606 & 49 & 631 & 77 & \\
30 & 123 & 153 & & 57 & 18 & 75 & 5 & 84 & 73 & \\
\hline Av. & 2643.4 & 427.2 & & 2314.6 & 77.7 & 2863.0 & 38.3 & & & \\
Av. ${ }^{\dagger}$ & 660.6 & 320.6 & & & & & & 549.0 & 101.1 & \\
Av. ${ }^{\dagger}$ & & & $4.63 \%$ & & & & & & & $4.01 \%$ \\
\hline
\end{tabular}




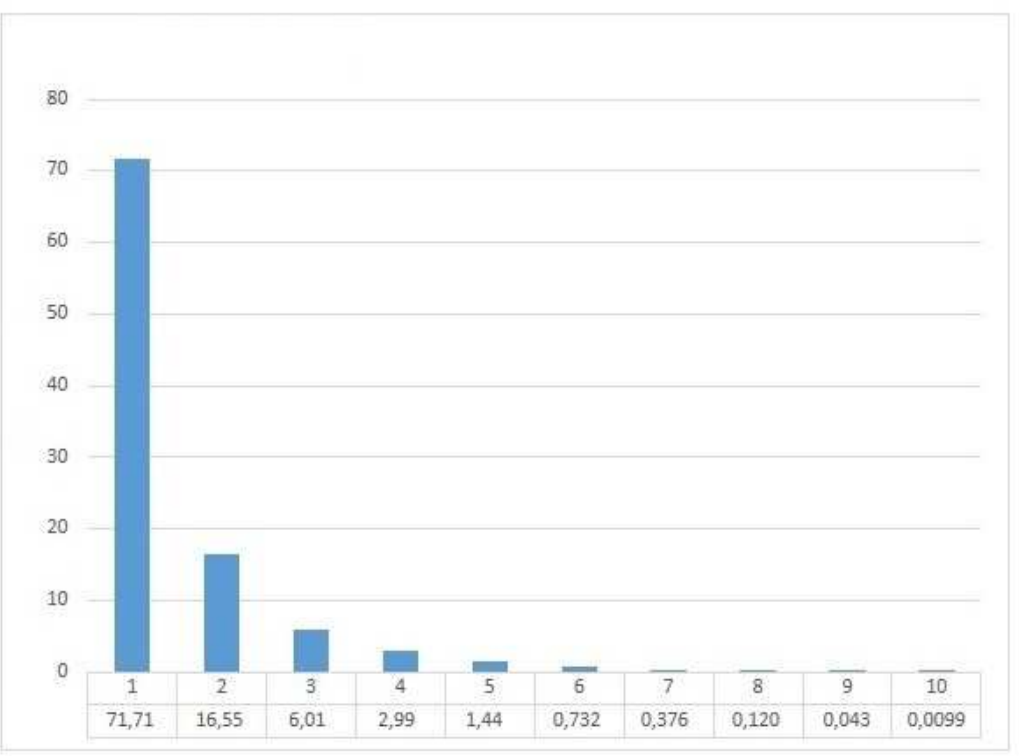

Fig. 3 Average of $\%$ of total triangular inequalities added

Table 2 Random instances with $n=34$ and $k=6$

\begin{tabular}{|c|c||cc|cc|cc|cc|}
\hline & Opt. & \multicolumn{2}{|c|}{ Strategy 1 } & \multicolumn{2}{c|}{ Strategy 2 } & \multicolumn{2}{c|}{ Strategy 3 } & \multicolumn{2}{c|}{ Strategy 4 } \\
N. & Value & Nodes Time & Nodes Time & Nodes Time & Nodes Time \\
\hline 1 & 3669.6 & 386 & 81 & 353 & 85 & 353 & 47 & 334 & 15 \\
2 & 3653.6 & 760 & 134 & 989 & 357 & 453 & 33 & 485 & 18 \\
3 & 3559.3 & 49 & 16 & 179 & 28 & 25 & 12 & 37 & 4 \\
4 & 3950.8 & 542 & 125 & 695 & 137 & 471 & 58 & 472 & 24 \\
5 & 3973.5 & 149 & 47 & 152 & 43 & 191 & 34 & 191 & 10 \\
6 & 3664.5 & 217 & 37 & 492 & 71 & 267 & 28 & 81 & 6 \\
7 & 3487.8 & 308 & 55 & 641 & 124 & 311 & 38 & 251 & 14 \\
8 & 3293.1 & 297 & 36 & 197 & 26 & 220 & 23 & 228 & 8 \\
9 & 3937.4 & 159 & 67 & 281 & 58 & 243 & 41 & 197 & 14 \\
10 & 3841.7 & 644 & 141 & 518 & 100 & 472 & 62 & 355 & 19 \\
\hline Av. & & 351.1 & 73.9 & 449.7 & 102.9 & 300.6 & 37.6 & $\mathbf{2 6 3 . 1}$ & $\mathbf{1 3 . 2}$ \\
\hline
\end{tabular}

Table 3 Random instances with $n=38$ and $k=7$

\begin{tabular}{|c|c||cc|cc|cc|cc|}
\hline & Opt. & \multicolumn{2}{|c|}{ Strategy 1 } & \multicolumn{2}{c|}{ Strategy 2 } & \multicolumn{2}{c|}{ Strategy 3 } & \multicolumn{2}{c|}{ Strategy 4 } \\
N. & Value & Nodes Time & Nodes Time & Nodes Time & Nodes Time \\
\hline 11 & 3344.4 & 188 & 100 & 30 & 33 & 80 & 29 & 18 & 7 \\
12 & 3456.8 & 498 & 272 & 618 & 117 & 115 & 23 & 124 & 8 \\
13 & 3361.7 & 284 & 133 & 213 & 56 & 278 & 46 & 220 & 14 \\
14 & 3646.4 & 709 & 286 & 395 & 68 & 384 & 45 & 288 & 14 \\
15 & 3384.7 & 1816 & 2434 & 1614 & 250 & 1469 & 188 & 1346 & 69 \\
16 & 3538.2 & 464 & 314 & 264 & 51 & 261 & 38 & 269 & 14 \\
17 & 3366.4 & 1834 & 1707 & 2455 & 485 & 2580 & 352 & 2962 & 198 \\
18 & 3565.2 & 360 & 218 & 213 & 42 & 134 & 32 & 67 & 11 \\
19 & 3029 & 100 & 73 & 301 & 39 & 317 & 37 & 148 & 11 \\
20 & 3846.6 & 1399 & 915 & 967 & 165 & 968 & 129 & 1038 & 63 \\
\hline Av. & & 765.2 & 645.2 & 707 & 130.6 & 658.6 & 91.9 & $\mathbf{6 4 8}$ & $\mathbf{4 0 . 9}$ \\
\hline
\end{tabular}


Table 4 Random instances with $n=44$ and $k=8$

\begin{tabular}{|c|c|c|c|c|c|c|c|}
\hline N. & $\begin{array}{l}\text { Opt. } \\
\text { Value }\end{array}$ & $\begin{array}{c}\text { Strategy } 1 \\
\text { Nodes Time }\end{array}$ & $\begin{array}{c}\text { Strategy } 2 \\
\text { Nodes Time }\end{array}$ & \multicolumn{2}{|c|}{$\begin{array}{c}\text { Strategy } 3 \\
\text { Nodes Time }\end{array}$} & \multicolumn{2}{|c|}{$\begin{array}{c}\text { Strategy } 4 \\
\text { Nodes Time }\end{array}$} \\
\hline 21 & 3706.1 & 624 & 445 & 421 & 127 & 322 & 46 \\
\hline 22 & 3896 & $4.65 \%$ & $3.37 \%$ & 2.5 & $5 \%$ & 11037 & 1721 \\
\hline 23 & 3692.7 & $936 \quad 977$ & $1058 \quad 302$ & 683 & 157 & 545 & 53 \\
\hline 24 & 3778.7 & $1756 \quad 2235$ & 1933 & 1784 & 508 & 1492 & 162 \\
\hline 25 & 4228 & $3.32 \%$ & $0.79 \%$ & 5083 & 2553 & 5413 & 894 \\
\hline 26 & 3844.3 & 808 & 276 & 677 & 171 & 574 & 67 \\
\hline 27 & 3731.2 & $4.06 \%$ & $5593 \quad 2318$ & 3943 & 1654 & 5360 & 721 \\
\hline 28 & 3567.7 & 53 & 114 & 43 & 49 & 50 & 21 \\
\hline 29 & 4205.5 & $1379 \quad 2576$ & 1312 & 1473 & 835 & 1518 & 262 \\
\hline 30 & 3893.2 & 175 & 165 & 135 & 68 & 123 & 33 \\
\hline$\overline{A v} .^{\top}$ & & 830.31090 & 840.4336 .1 & 745.1 & 273.6 & 660.6 & 92 \\
\hline
\end{tabular}

Table 5 Random instances with $n=48$ and $k=9$

\begin{tabular}{|c|c||cc|cc|cc|cc|}
\hline & Opt. & \multicolumn{2}{|c|}{ Strategy 4} & \multicolumn{2}{c|}{+ 2-partition } & \multicolumn{2}{|c|}{+ Weight-Cover } & \multicolumn{2}{|c|}{+ Both } \\
N. & Value & Nodes Time & Nodes & Time & Nodes & Time & Nodes & Time \\
\hline 31 & 3909.5 & 2985 & 386 & 1857 & 311 & 2773 & 368 & 1665 & 298 \\
32 & 3884.7 & 213 & 52 & 243 & 58 & 265 & 51 & 232 & 52 \\
33 & 3792.7 & 3376 & 329 & 2372 & 296 & 3221 & 295 & 2296 & 272 \\
34 & 4264.9 & 1945 & 622 & 2092 & 659 & 1986 & 478 & 2097 & 588 \\
35 & 3785.3 & 525 & 113 & 453 & 115 & 449 & 80 & 425 & 80 \\
36 & 3701.5 & 217 & 87 & 198 & 86 & 243 & 64 & 158 & 57 \\
37 & 4000.5 & 2542 & 729 & 2406 & 512 & 1234 & 234 & 1633 & 267 \\
38 & 4885.9 & 10132 & 2038 & 6226 & 2055 & 7294 & 1652 & 4902 & 1233 \\
39 & 3895.8 & 4980 & 1295 & 2784 & 1034 & 3956 & 852 & 3552 & 884 \\
40 & 3627.2 & 122 & 65 & 115 & 49 & 100 & 45 & 72 & 43 \\
\hline Av. & & 2703.7 & 571.6 & 1874.6 & 517.5 & 2152.1 & 411.9 & $\mathbf{1 7 0 3 . 2} \mathbf{3 7 7 . 4}$ \\
\hline
\end{tabular}

Table 6 Random instances with $n=54$ and $k=10$

\begin{tabular}{|c|c|c|c|c|c|c|}
\hline N. & $\begin{array}{c}\text { Opt. } \\
\text { Value }\end{array}$ & $\begin{array}{c}\text { Strategy } 4 \\
\text { Nodes Time }\end{array}$ & $\begin{array}{l}+2 \text {-partition } \\
\text { Nodes Time }\end{array}$ & $\begin{array}{l}+ \text { Weight-Cover } \\
\text { Nodes Time }\end{array}$ & \multicolumn{2}{|c|}{+ Both } \\
\hline 41 & & $2.33 \%$ & $2.29 \%$ & $2.34 \%$ & \multicolumn{2}{|c|}{$1.77 \%$} \\
\hline 42 & 3891.9 & 1673 & 1770 & 782 & 1931 & 767 \\
\hline 43 & 4153 & $7688 \quad 3221$ & $6336 \quad 3309$ & 7490 & 6409 & 2402 \\
\hline 44 & & $2.06 \%$ & $2.05 \%$ & $1.56 \%$ & \multicolumn{2}{|c|}{$1.79 \%$} \\
\hline 45 & 4321.1 & 1916 & 1869 & 3305 & 2570 & 578 \\
\hline 46 & & $1.67 \%$ & $2.10 \%$ & $1.65 \%$ & \multicolumn{2}{|c|}{$1.62 \%$} \\
\hline 47 & 4203.2 & 3519 & 3043 & 2869 & 4824 & 2084 \\
\hline 48 & 4039.1 & $1.31 \%$ & $1.60 \%$ & $1.87 \%$ & 7797 & 3173 \\
\hline 49 & 4270.9 & 3337 & 1017 & 2865 & 2716 & 664 \\
\hline 50 & 3916.5 & 1640 & 1654 & 3977 & 3529 & 1247 \\
\hline $\mathrm{Av} .^{\dagger}$ & & 5166.22011 .0 & 3998.21963 .5 & $\begin{array}{ll}4187.5 & 1648.7\end{array}$ & 3663.2 & 1290.3 \\
\hline
\end{tabular}

